\title{
CONTINUOUS PROJECTIONS ONTO IDEAL CONVERGENT SEQUENCES
}

\author{
PAOLO LEONETTI
}

\begin{abstract}
Let $\mathcal{I} \subseteq \mathcal{P}(\omega)$ be a meager ideal. Then there are no continuous projections from $\ell_{\infty}$ onto the set of bounded sequences which are $\mathcal{I}$-convergent to 0 . In particular, it follows that the set of bounded sequences statistically convergent to 0 is not isomorphic to $\ell_{\infty}$.
\end{abstract}

\section{INTRODUCTION}

A closed subspace $X$ of a Banach space $B$ is said to be complemented in $B$ if there exists a continuous projection from $B$ onto $X$. It is known that $c_{0}$, the space of real sequences convergent to 0 , is not complemented in $\ell_{\infty}$, cf. [10, 12]. The aim of this note is to show the ideal analogue of this result.

Let $\mathcal{I} \subseteq \mathcal{P}(\omega)$ be an ideal, that is, a family closed under subsets and finite unions. It is also assumed that Fin $:=[\omega]^{<\omega} \subseteq \mathcal{I}$ and $\omega \notin \mathcal{I}$. Set $\mathcal{I}^{+}:=\mathcal{P}(\omega) \backslash \mathcal{I}$. In particular, each $\mathcal{I}$ can be regarded as a subset of the Cantor space $2^{\omega}$ with the product topology, so we can speak of Borel ideals, $F_{\sigma}$ ideals, etc. An ideal $\mathcal{I}$ is said to be a P-ideal if it is $\sigma$-directed modulo finite sets, i.e., for each sequence $\left(A_{n}\right)$ in $\mathcal{I}$ there exists $A \in \mathcal{I}$ such that $A_{n} \backslash A$ is finite for all $n \in \omega$. We refer to [7] for a recent survey on ideals and filters.

A real sequence $\left(x_{n}\right)$ is said to be $\mathcal{I}$-convergent to $y$ if $\left\{n: x_{n} \notin U\right\} \in \mathcal{I}$ for all neighborhoods $U$ of $y$. We denote by $c(\mathcal{I})\left[\right.$ resp. $\left.c_{0}(\mathcal{I})\right]$ the space of real sequences which are $\mathcal{I}$-convergent [resp. $\mathcal{I}$-convergent to 0$]$. The set of bounded real $\mathcal{I}$ convergent sequences has been studied, e.g., in $[2,6,8]$. By an easy modification of [8, Theorem 2.3], $c_{0}(\mathcal{I}) \cap \ell_{\infty}$ is a closed linear subspace of $\ell_{\infty}$ (with the sup norm).

The question addressed here, posed at the open problem session of the 45th Winter School in Abstract Analysis (Czech Republic, 2017), follows:

Question 1. Is $c_{0}(\mathcal{I}) \cap \ell_{\infty}$ complemented in $\ell_{\infty}$ ?

Before proving our main result, we recall the following:

2010 Mathematics Subject Classification. Primary: 40A35, 46B03. Secondary: 54A20, $46 \mathrm{~B} 26$.

Key words and phrases. Meager ideal, $\mathcal{I}$-maximal almost disjoint family, complementability, asymptotic density zero sets, $\mathcal{I}$-convergent sequence. 
Lemma 1.1. An infinite dimensional subspace $X$ of $\ell_{\infty}$ is complemented in $\ell_{\infty}$ if and only if it is isomorphic to $\ell_{\infty}$.

Proof. See [1, Proposition 2.5.2 and Theorem 5.6.5].

Hence, Question 1 can be reformulated as:

Question 2. Is $c_{0}(\mathcal{I}) \cap \ell_{\infty}$ isomorphic to $\ell_{\infty}$ ?

We will prove that the answer is negative for a large class of ideals. To state our result, we recall that a family $\mathscr{A} \subseteq \mathcal{I}^{+}$is said to be $\mathcal{I}$-maximal-almost-disjoint (in short, $\mathcal{I}$-mad) if $\mathscr{A}$ is a maximal family (with respect to inclusion) such that $A \cap B \in \mathcal{I}$ for all distinct $A, B \in \mathscr{A}$, so that for each $X \in \mathcal{I}^{+}$there exists $A \in \mathscr{A}$ such that $X \cap A \in \mathcal{I}^{+}$. (The minimal cardinality $\mathfrak{a}(\mathcal{I})$ of an $\mathcal{I}$-mad has been studied in the literature: e.g., it is known that, if $\mathcal{I}$ is an analytic P-ideal, $\mathfrak{a}(\mathcal{I})>\omega$ if and only if $\mathcal{I}$ is $F_{\sigma}$, cf. $[4,5]$.)

Our main result follows:

Theorem 1.2. Let $\mathcal{I}$ be an ideal for which there exists an uncountable $\mathcal{I}$-mad family. Then $c_{0}(\mathcal{I}) \cap \ell_{\infty}$ is not complemented in $\ell_{\infty}$.

It can be shown that, if $\mathcal{I}$ is a meager ideal, there is an $\mathcal{I}$-mad family of cardinality $\mathfrak{c}$, see Lemma 2.3 below. In particular

Corollary 1.3. $c_{0}(\mathcal{I}) \cap \ell_{\infty}$ is not complemented in (and not isomorphic to) $\ell_{\infty}$ whenever $\mathcal{I}$ is meager.

As an important example, the family of asymptotic density zero sets $\mathcal{Z}:=\{S \subseteq$ $\omega:|S \cap[1, n]| / n \rightarrow 0\}$ is an analytic P-ideal, hence meager. Therefore:

Corollary 1.4. The set of bounded real sequences statistically convergent to 0 (i.e., $c_{0}(\mathcal{Z})$ ) is not is isomorphic to $\ell_{\infty}$.

Lastly, we obtain an analogue of the main result in [9] (for summability matrices):

Corollary 1.5. $c$ is complemented in $c(\mathcal{I}) \cap \ell_{\infty}$ if and only if $\mathcal{I}=$ Fin.

It is worth noting that Theorem 1.2 cannot be extended to all ideals $\mathcal{I}$. Indeed, if $\mathcal{I}$ is maximal, then the set of bounded $\mathcal{I}$-convergent sequences, which is isomorphic to $c_{0}(\mathcal{I}) \cap \ell_{\infty}$, is exactly $\ell_{\infty}$.

\section{Preliminaries And Proofs}

Thanks to Lemma 1.1, a negative question to Question 1 would follow if $c_{0}(\mathcal{I}) \cap$ $\ell_{\infty}$ was separable (indeed $\ell_{\infty}$ is nonseparable, hence they cannot be isomorphic). However, this works only if $\mathcal{I}=$ Fin:

Lemma 2.1. $c_{0}(\mathcal{I})$ is separable if and only if $\mathcal{I}=$ Fin. 
Proof. The if part is known. Conversely, let us suppose that there exists $A \in$ $\mathcal{I} \cap[\omega]^{\omega}$. For each $X \subseteq \omega$ and $\varepsilon>0$, let $B\left(\mathbf{1}_{X}, \varepsilon\right)$ be the open ball with center $\mathbf{1}_{X}$ and radious $\varepsilon$. The collection $\mathscr{B}:=\left\{B\left(\mathbf{1}_{X}, 1 / 2\right): X \in[A]^{\omega}\right\}$ is an uncountable family of nonempty open sets which are pairwise disjoint, hence $c_{0}(\mathcal{I})$ is not separable.

At this point, recall the following characterization, see [11] and [3, Theorem 4.1.2]:

Lemma 2.2. $\mathcal{I}$ is a meager ideal if and only if there exists a finite-to-one function $f: \omega \rightarrow \omega$ such that $f^{-1}(A) \in \mathcal{I}$ if and only if $A$ is finite.

In other words, the second condition is Fin $\leq_{\mathrm{RB}} \mathcal{I}$, where $\leq_{\mathrm{RB}}$ is the RudinBlass ordering. This is sufficient to prove the existence of an uncountable $\mathcal{I}$-mad family:

Lemma 2.3. There exists an $\mathcal{I}$-mad family of cardinality $\mathfrak{c}$, provided $\mathcal{I}$ is meager.

Proof. It is known that there is a Fin-mad family $\mathscr{A}$ of cardinality $\mathfrak{c}$, cf. [12]. Then, thanks to Lemma 2.2, there exists a finite-to-one function $f: \omega \rightarrow \omega$ such that $f^{-1}(A) \in \mathcal{I}$ if and only if $A$ is finite, hence $\left\{f^{-1}(A): A \in \mathscr{A}\right\}$ is the claimed $\mathcal{I}$-mad family.

Let us prove our main result:

Proof of Theorem 1.2. Let us suppose for the sake of contradiction that $c_{0}(\mathcal{I}) \cap \ell_{\infty}$ is complemented in $\ell_{\infty}$ and denote by

$$
\pi: \ell_{\infty} \rightarrow c_{0}(\mathcal{I}) \cap \ell_{\infty}
$$

the canonical projection. Define $T:=I-\pi$, hence $T$ is bounded linear operator such that $T(x)=0$ for each $x \in c_{0}(\mathcal{I}) \cap \ell_{\infty}$. Note also that, if $B \notin \mathcal{I}$, then $\mathbf{1}_{B}$ is a bounded sequence which is not $\mathcal{I}$-convergent to 0 , hence $\pi\left(\mathbf{1}_{B}\right) \neq \mathbf{1}_{B}$ and $T\left(\mathbf{1}_{B}\right) \neq 0$.

At this point, let $\left(A_{j}: j \in J\right)$ be an uncountable $\mathcal{I}$-mad family, which exists by hypothesis. We are going to show that there exists $j \in J$ such that $T\left(\mathbf{1}_{A_{j}}\right)=0$, which is impossible since $A_{j} \in \mathcal{I}^{+}$. Indeed, let us suppose that, for each $j \in J$, there exists $x_{j}=\left(x_{j, n}\right) \in \ell_{\infty}$ supported on $A_{j}$ with $T\left(x_{j}\right) \neq 0$ and, without loss of generality, $\left\|x_{j}\right\|_{\infty}=1$. It follows that there exists $m, k \in \omega$ such that $\tilde{J}:=\left\{j \in J:\left|x_{j, m}\right| \geq 2^{-k}\right\}$ is uncountable. Also, by possibly replacing $x_{j}$ with $-x_{j}$, let us suppose without loss of generality that $x_{j, m}>0$ for all $j \in \tilde{J}$.

For each nonempty finite set $F \subseteq \tilde{J}$, define $s_{F}=\left(s_{F, n}\right):=\sum_{j \in F} x_{j}$. In particular,

$$
\left\|T\left(s_{F}\right)\right\|_{\infty} \geq s_{F, m} \geq|F| 2^{-k} .
$$

Note also that $I:=\bigcup\left(A_{i} \cap A_{j}\right)$, where the sum is extended over all distinct $i, j \in F$, belongs to $\mathcal{I}$. This implies that the sequence $s_{F} \uparrow I$ is $\mathcal{I}$-convergent to 0 , 
hence $T\left(s_{F}\right)=T\left(s_{F}\left\lceil I^{c}\right)\right.$. Therefore

$$
\left\|T\left(s_{F}\right)\right\|_{\infty}=\| T\left(s _ { F } \lceil I ^ { c } ) \| _ { \infty } \leq \| T \| \cdot \| s _ { F } \left\lceilI^{c}\left\|_{\infty} \leq\right\| T \|,\right.\right.
$$

which, together with (1), implies $|F| \leq 2^{k}\|T\|$. This contradicts the fact the $\tilde{J}$ is infinite.

Proof of Corollary 1.5. There is nothing to prove if $\mathcal{I}=$ Fin. Conversely, fix $I \in \mathcal{I} \backslash$ Fin and define $X:=\left\{x \in \ell_{\infty}: x_{i} \neq 0\right.$ only if $\left.i \in I\right\}$ and $Y:=X \cap c_{0}$. It is clear that

$$
c \subseteq Y \subseteq X \subseteq c(\mathcal{I}) \cap \ell_{\infty}
$$

and that $X$ and $Y$ are isometric to $\ell_{\infty}$ and $c_{0}$, respectively. Hence, it is known that $c$ can be projected continuously onto $Y$, let us say through $T$, see [10]. To conclude the proof, let us suppose that there exists a continuous projection $H: c(\mathcal{I}) \cap \ell_{\infty} \rightarrow c$. Then the restriction $T \circ H \uparrow X$ is a continuous projection $\ell_{\infty} \rightarrow c_{0}$. This contradicts Theorem 1.2 (in the case $\mathcal{I}=$ Fin).

2.1. Acknowledgments. The author is grateful to Tommaso Russo (Università degli Studi di Milano, IT) for suggesting Question 1 and Lemma 1.1.

\section{REFERENCES}

1. F. Albiac and N. J. Kalton, Topics in Banach space theory, Graduate Texts in Mathematics, vol. 233, Springer, New York, 2006.

2. A. Bartoszewicz, S. Głab, and A. Wachowicz, Remarks on ideal boundedness, convergence and variation of sequences, J. Math. Anal. Appl. 375 (2011), no. 2, 431-435.

3. T. Bartoszyński and H. Judah, Set theory, A K Peters, Ltd., Wellesley, MA, 1995, On the structure of the real line.

4. J. E. Baumgartner, Iterated forcing, Surveys in set theory, London Math. Soc. Lecture Note Ser., vol. 87, Cambridge Univ. Press, Cambridge, 1983, pp. 1-59.

5. B. Farkas and L. Soukup, More on cardinal invariants of analytic P-ideals, Comment. Math. Univ. Carolin. 50 (2009), no. 2, 281-295.

6. R. Filipów and J. Tryba, Ideal convergence versus matrix summability, Studia Math., to appear.

7. M. Hrušák, Combinatorics of filters and ideals, Set theory and its applications, Contemp. Math., vol. 533, Amer. Math. Soc., Providence, RI, 2011, pp. 29-69.

8. P. Kostyrko, M. Mačaj, T. Šalát, and M. Sleziak, $\mathscr{I}$-convergence and extremal $\mathscr{I}$-limit points, Math. Slovaca 55 (2005), no. 4, 443-464.

9. J. Lindenstrauss, Mathematical Notes: A Remark Concerning Projections in Summability Domains, Amer. Math. Monthly 70 (1963), no. 9, 977-978.

10. A. Sobczyk, Projection of the space $(m)$ on its subspace $\left(c_{0}\right)$, Bull. Amer. Math. Soc. 47 (1941), 938-947.

11. M. Talagrand, Compacts de fonctions mesurables et filtres non mesurables, Studia Math. 67 (1980), no. 1, 13-43.

12. R. Whitley, Mathematical Notes: Projecting $m$ onto $c_{0}$, Amer. Math. Monthly 73 (1966), no. $3,285-286$. 
Department of Statistics, Università "L. Bocconi", via Roentgen 1, 20136 Milan, ITALY

E-mail address: leonetti.paolo@gmail.com

URL: https://sites.google.com/site/leonettipaolo/ 